\title{
Nanoliposomal L-Asparaginase and Its Antitumor Activities in Lewis Lung Carcinoma Tumor-Induced BALB/c Mice
}

\author{
Thi Thao Do $\mathbb{D}^{1,2}$ Thi Phuong Do, ${ }^{1}$ Thi Nga Nguyen, ${ }^{1}$ Thi Cuc Nguyen, ${ }^{1}$ \\ Thi Thu Phuong Vu, ${ }^{1}$ and Thi Giang An Nguyen ${ }^{3}$ \\ ${ }^{1}$ Institute of Biotechnology, Vietnam Academy of Science and Technology, 18 Hoang Quoc Viet Road, Nghia Do, Cau Giay, \\ Ha Noi, Vietnam \\ ${ }^{2}$ Graduate University of Science and Technology, Vietnam Academy of Science and Technology, 18 Hoang Quoc Viet Road, \\ Nghia Do, Cau Giay, Ha Noi, Vietnam \\ ${ }^{3}$ School of Natural Sciences Education, Vinh University, 182 Le Duan Str, Vinh, Nghe An Province, Vietnam \\ Correspondence should be addressed to Thi Thao Do; thaodo74@yahoo.com
}

Received 14 January 2019; Revised 15 March 2019; Accepted 18 March 2019; Published 2 May 2019

Guest Editor: Dinh Quang Khieu

Copyright (C) 2019 Thi Thao Do et al. This is an open access article distributed under the Creative Commons Attribution License, which permits unrestricted use, distribution, and reproduction in any medium, provided the original work is properly cited.

\begin{abstract}
Although L-Asparaginase (L-ASP) is an effective chemotherapeutic agent, it has side effects such as fever, skin rashes, chills, anaphylaxis, and severe allergic reactions. Moreover, the short half-life of L-ASP reduces its antitumor activity. To reduce its side effects and broaden its pharmaceutical applications, L-ASP obtained from Pectobacterium carotovorum was subjected to liposomal conjugation. The enzyme was then loaded into liposomes using the hydrated thin-film method. The in vitro cytotoxic activity of liposomal L-ASP was evaluated with the MTT assay using cancerous cell lines, and its antitumor effects were examined in Lewis lung carcinoma (LLC) tumorized mice. The average size of the liposomes containing purified L-asparagine was $93.03 \pm 0.49 \mathrm{~nm}$. They had a zeta potential of $-15.45 \pm 6.72 \mathrm{mV}$, polydispersity index of $0.22 \pm 0.02$, and encapsulation efficiency of $53.99 \pm 5.44 \%$. The in vitro cytotoxic activity of liposomal L-ASP was less effective against LLC, MCF-7 (human breast carcinoma), HepG2 (human hepatocellular carcinoma), SK-LU-1 (human lung carcinoma), and NTERA-2 (pluripotent human embryonic carcinoma) cells than that of free L-ASP. However, the antitumor activity of liposomal L-ASP was significantly greater than that of untrapped L-ASP at the same doses (6 UI/mouse) in terms of tumor size $\left(6309.11 \pm 414.06 \mathrm{~mm}^{3}\right)$ and life span (35.00 \pm 1.12 days). This is the first time the antitumor activities of PEGylated nanoliposomal L-ASP have been assessed in LLC carcinoma tumorinduced $\mathrm{BALB} / \mathrm{c}$ mice and showed significantly improved pharmacological properties compared to those of free L-ASP $(P<0.05)$. Thus, nanoliposomal L-ASP should be considered for its widening applications against carcinoma tumors.
\end{abstract}

\section{Introduction}

L-Asparaginase (L-ASP) hydrolyzes L-asparagine into Laspartate and ammonia and is used to treat acute lymphoblastic leukemia (ALL) and non-Hodgkin lymphoma [1]. Although the enzyme has been a mainstay in the treatment of ALL [2-4], it can induce fever, skin rashes, chills, anaphylaxis, and severe allergic reactions $[5,6]$. Continued treatment leads to frequent hypersensitivity reactions in the enzyme as a foreign protein $[7,8]$. To minimize the incidence of systemic immunological reactions and other limitations of L-ASP, liposomal encapsulation of the enzyme using different techniques has been adopted. The main components of liposomes are phospholipids, which are similar to cell membranes [9]. The phospholipids form a bilayer membrane, which allows drugs with different physicochemical properties to be loaded into the liposome or conjugated into the biolayers and then delivered to a lesion [10]. When liposomes are loaded with enzymes, they can be targeted to organs such as the spleen, liver, and bone marrow [11], prolonging the circulation time without inhibiting enzymatic activities [12]. L-ASP was clarified for its antitumor activities in breast tumor bearing mice by Shiromizu et al. [13]. However, liposomal L-ASP has been 
only reported for its improvement in vivo anticancer activities in lymphomatic mice [11]. Therefore, we developed PEGylated nanoliposomal L-ASP and first time evaluated its antitumor efficacy in Lewis lung carcinoma (LLC) tumorized $\mathrm{BALB} / \mathrm{c}$ mice for broadening its pharmaceutical applications.

\section{Materials and Methods}

2.1. Materials. The bacterial strain Pectobacterium carotovorum was provided by Prof. Gilles Truan (Axe Biocatalyse/Ingénieries Métabolique et Moléculaire, LISBP, INSA, Toulouse, France). The strain was used for the production of L-ASP enzyme. 1,2-Distearoyl-sn-glycero-3phosphoethanolamine-N-[amino(polyethylene glycol)-2000] (DSPE-PEG-2000) was purchased from Avanti polar lipids Inc. (Alabaster, Alabama, USA). Cholesterol, soybean lecithin, fetal bovine serum (FBS), and gentamicin was obtained from Sigma Chemical Co. (St. Louis, MO., USA). Dulbecco's modified Eagle's medium and nonessential amino acid (NAA) and L-glutamine was purchased from Invitrogen (Carlsbad, CA, USA).

2.2. Animals. Male and female albino BALB/c mice (810 weeks old) were obtained from the Institute of Biotechnology, Vietnam Academy of Science and Technology (VAST, Hanoi, Vietnam). All mice were housed in a temperature-controlled room on a 12-hour light/12-hour dark cycle with food and water ad libitum. Experiments were performed in accordance with Vietnamese Ethical Laws and European Communities Council Directives of November 24, 1986 (86/609/EEC) guidelines for the care and use of laboratory animals.

\subsection{Isolation, Extraction, and Purification of L-Asparaginase.} The bacterium was cultivated in the optimal medium to produce L-asparaginase as reported by Gulati et al. [14]. In details, the inoculum was prepared by adding a loop full of $250 \mathrm{ml}$ cultivated medium in a $1 \mathrm{~L}$ flask and incubated at $30^{\circ} \mathrm{C}, 180 \mathrm{rpm}$ in a shaking incubator for $12 \mathrm{~h}$ (to reach the culture OD at $600 \mathrm{~nm}=0.6$ to 0.8 ). The continued $2 \%$ of the inoculum was continuously incubated in a shaking incubator at $30^{\circ} \mathrm{C}, 180 \mathrm{rpm}$ for additional $24 \mathrm{~h}$. Cells were harvested by centrifugation at $10,000 \mathrm{~g}$ for $10 \mathrm{~min}$ at $4^{\circ} \mathrm{C}$. Cells were washed with $50 \mathrm{mM}$ Tris- $\mathrm{HCl}$ buffer $(\mathrm{pH} 8,6)$ and resuspended in the same buffer. The cells were cooled on ice and ultrasonicated at $20 \mathrm{MHz}, 35 \%$ amplitude, $20 \mathrm{~min}$. The lysate was centrifuged at $20,000 \mathrm{~g}$ for $10 \mathrm{~min}$ at $4^{\circ} \mathrm{C}$. The clear supernatant was loaded on DEAE cellulose and Sephadex G-100 chromatography for purification. The obtained eluted fractions were protein-quantitated using Pierce BCA Protein Assay Kit (Thermo Scientific) and qualified using SDSPAGE [15].

2.4. Determination of L-Asparaginase Activity. L-ASP activity was measured by the modified method of Wriston [16] in which the rate of ammonia formation will be detected by Nessler' reagent at $37^{\circ} \mathrm{C}$. One unit of L-ASP activity was defined that liberates the amount of enzyme that released $1 \mu \mathrm{M}$ of ammonia (with $10 \mu \mathrm{M}-10 \mathrm{mM}$ ammonium sulfate as the standard) per minute under the assay conditions.

2.5. Procedure for the Preparation of L-ASP Nanoliposomes. L-ASP was entrapped into liposomes by thin film dehydration-rehydration method, as Cruz et al. [17] with slight modification. Briefly, soybean lecithin $(40 \mu \mathrm{mol})$, cholesterol $(4 \mu \mathrm{mol})$, and DSPE-PEG-2000 ( $8 \mu \mathrm{mol})$ were dissolved in chloroform:methanol $(9: 1 \mathrm{v} / \mathrm{v})$ and stirred mechanically to form homogeneous mixture in round bottom flask. The solvent was removed using a rotary evaporator under an aspirate vacuum $(25 \mathrm{mmHg})$ and a water bath with the temperature maintained at $25^{\circ} \mathrm{C}$. The thin film which formed on the walls of the flask was dispersed in $2 \mathrm{ml}$ of $5 \mathrm{mM}$ potassium phosphate buffer ( $\mathrm{pH} 7.5$ ) containing L-ASP (150 I.U.) and rotated without vacuum at $100 \mathrm{rpm}, 25^{\circ} \mathrm{C}$. Then, the multilamellar vesicles were sonicated three times at 30 seconds intervals for resizing before filtered through $0.22 \mu \mathrm{m}$ membrane to receive the L-ASP liposomal mixture. Finally, the liposomal mixture was washed twice with normal saline.

The particle size, zeta potentials, and size distribution of liposomes were determined using a Zetasizer Nano-Z (Malvern Instruments, UK). The conjugates were also observed by high-solution transmission electronic microscopy (TEM) (Jeol 1200EX TEM, Jeol Company, Tokyo, Japan). The liposomal samples were mounted on metal stands and coated with gold to thickness of 200-500 $\mathrm{A}^{\circ}$. Then, the plates were magnified $200 \mathrm{x}$ to capture the morphology of the prepared liposomes.

2.6. Determination of Encapsulation Efficiency (EE). After preparation, liposomal solution was loaded to Vivaspin column and centrifuged at $3000 \mathrm{RCF}$ for $20 \mathrm{~min}, 25^{\circ} \mathrm{C}$ to remove the free L-ASP. The liposome pellet was lysed with $10 \%$ Triton X-100 to disrupt the liposomal bilayer and to release L-ASP. The Bradford assay was used to determine the amount of released L-ASP. Encapsulation efficiency of L-ASP into liposomes is determined as the following formula [18]:

$$
\mathrm{EE} \%=\frac{C_{\mathrm{f}}}{C_{\mathrm{i}}} \times 100,
$$

where $C_{\mathrm{f}}$ is the encapsulated amount of L-ASP into liposomes measured after lysing with $10 \%$ Triton-X 100 and $C_{\mathrm{i}}$ is the L-ASP amount added to the lipid mixture.

\subsection{Antiproliferative Activities of L-ASP Encapsulated} Nanoliposomes. The antiproliferative assays were carried out in triplicate in 96-well microtiter plates against LLC (Lewis lung carcinoma), MCF-7 (human breast carcinoma), HepG2 (human hepatocellular carcinoma), SKLU-1 (human lung carcinoma), and NTERA-2 (pluripotent human embryonic carcinoma) cells. Those cells were maintained in Dulbecco's modified Eagle's medium supplemented with 10\% FBS (Sigma-Aldrich, St. Louis, MO), 
$1 \%$ antibiotic-antimycotic (Thermo Fisher), 1\% nonessential amino acid, and $2 \mathrm{mM}$ of L-glutamine. For MCF-7, insulin was added to the growth medium at $10 \mu \mathrm{g} / \mathrm{ml}$ concentration. All cells were incubated in a humidifier with $5 \% \mathrm{CO}_{2}$ at $37^{\circ} \mathrm{C}$ and subcultured every 2-3 days. The cancer cells were seeded into a 96-well plate at a density of $1 \times 10^{4}$ cells/well. Then nanoliposomal conjugates were added to reach the final concentration ranging from $0.01-2.5 \mathrm{UI} / \mathrm{mL}$ and incubated for further $72 \mathrm{~h}$. To assess effect of liposomes on cell viability, the MTT assay was performed as previously described [19].

2.8. Antitumor Efficiency of L-ASP Encapsulated Liposomes. The experiment was carried out using BALB/c mice at 20-25 gr weight. Mice were inoculated by subcutaneously injection with $1 \times 10^{6}$ LLC cells to induce tumor. On the day $7^{\text {th }}$ of LLC cell injection, mice were randomly divided into 4 groups (6 mice per group) including a control group, two treated groups of liposomal L-ASP $(6 \mathrm{UI} /$ mouse and $3 \mathrm{UI} /$ mouse), and the free L-ASP treated group (6 UI/ mouse). The drugs were administrated intravenously every 2 days. Growth of tumor was determined by caliper measurements in two dimensions, length $(L)$ and width $(W)$, every 7 days. The tumor volume $(V)$ was calculated using the formula $V=1 / 2 \times L \times W^{2}$ [20].

The survival time of mice in all experimental groups were recorded. It was calculated from the day of LLC cell inoculation to the day of death, and percentage increase in average life span (ILS) was calculated by the formula \% ILS $=(A / B-1) \times 100$ in which $A$ means survival time of treated, $B$ is mean survival time of control group, and ILS is increase in average life span group.

2.9. Statistical Analysis. Statistical analyses and significance, as measured by two-way analysis of variance (ANOVA), were performed using GraphPad PRISM 5.0 software (GraphPad Software, USA). In all comparisons, $P<0.05$ was considered statistically significance.

\section{Results and Discussion}

3.1. Isolation, Purification, and Determination of L-ASP Activity. First, L-ASP crude extract was purified in three steps with a final yield of $23.5 \%$ and a purification fold of 9.38 (Table 1). The estimated molecular weight of the obtained enzyme was $36 \mathrm{kDa}$ (Figure 1). This purified enzyme was used for further liposomal conjugation.

\subsection{Physicochemical Characteristics of Liposomal L-ASP.} Generally, the components of a bilayer have strong effects on the rigidity, fluidity, and toxicity of the phospholipid bilayer [21]. Nontoxic phospholipids are usually selected to reduce toxicity and to strengthen drug-liposomal conjugates, including natural and synthetic phospholipids such as soybean lecithin and DSPE-PEG-2000 [10]. Soybean lecithin is a natural phospholipid that is often used for large-scale industrial applications to reduce production costs [22]. DSPEPEG-2000 is reported elsewhere as important breakthrough in the liposomal development pertaining stealth behavior [23]. Cholesterol is often used in liposomal formulation because it facilitates complex interactions with phospholipids and other lipids in cellular membranes [24]. Therefore, we used soybean lecithin, DSPE-PEG-2000, and cholesterol to make liposomal L-ASP, encapsulating the purified L-ASP using the rehydrated thin film method. The resulting liposomes were $93.03 \pm 0.49 \mathrm{~nm}$ in size with a polydispersity index of $0.22 \pm 0.02$, indicating that the size distribution was quite homogeneous. The zeta potential is a measure of the magnitude of electrostatic or charge repulsion between particles, which affects liposome stability [25-27]. The liposomes harboring L-ASP had a zeta potential of $-15.45 \pm 6.72 \mathrm{mV}$, the opposite of liposomes reported by Bahreini et al. that were made from chitosan and tripolyphosphate using ionotropic gelation [28]. The entrapment efficiency was determined as the ratio of encapsulated L-ASP to the amount used to prepare the liposomes. The encapsulation efficiency was $53.99 \%$, demonstrating that L-ASP was effectively loaded into the liposomes (Table 2).

3.3. High-Resolution Transmission Electron Microscopic Analysis. High-resolution transmission electron microscope (TEM) was used to evaluate the size, shape, and morphology of the L-ASP liposomes [26]. On TEM, the LASP liposomal particles were spherical and evenly dispersed (Figure 2). The obtained liposomes were nanometers in size (Figure 2).

3.4. Cytotoxicity Activities. The effects of free and liposomal L-ASP on the survival of LLC (Lewis lung carcinoma), MCF7 (human breast carcinoma), HepG2 (human hepatocellular carcinoma), SK-LU-1 (human lung carcinoma), and NTERA-2 (pluripotent human embryonic carcinoma) cell lines were evaluated using the MTT assay. The cytotoxic activity of the liposomal L-ASP was dependent on the cancer cell line and concentrations tested (Table 3 and Figure 3). SK-LU-1 cells were the most sensitive to liposomal L-ASP and to the unloaded enzyme. The conjugates had similar activity on SK-LU-1 and LLC cells, with $\mathrm{IC}_{50}$ values of $0.21 \pm 0.03$ and $0.23 \pm 0.02 \mathrm{UI} / \mathrm{mL}$, respectively. MCF-7 cells were the least sensitive to both free and liposomal L-ASP. A decrease in the L-ASP concentration led to reduced cell death in all cell lines tested. Generally, malignant cells have a high division rate and need more nutrients than normal cells. Asparagine is important in cell growth and function, as it coordinates protein and nucleotide synthesis [29]. L-ASP induces cytotoxicity by breaking asparagine into L-aspartate and ammonia. According to Moharib, the cytotoxic activity of L-ASP is closely related to asparagine, and the asparagine level differed among various cell lines [30]. Therefore, L-ASP induced cancer cell death to varying degrees. However, the cytotoxic activity of the liposomal L-ASP was 3.00-5.68 times lower than that of free L-ASP after $72 \mathrm{~h}$ of treatment. In general, and with L-ASP, liposomal formulations are significantly less toxic than the unconjugated forms because of slow uptake. In our study, the L-ASP liposomes were less active against all cancer cells, in agreement with other studies 
TABle 1: Purification profile of isolated L-ASP.

\begin{tabular}{lccccc}
\hline Steps & Total activity (IU) & Total protein $(\mathrm{mg})$ & Specific activity (IU/mg) & Purification fold & Yield (\%) \\
\hline Crude extract & 5972 & 276.5 & 21.6 & 0 & 100 \\
Ammonium sulfate precipitation & 2204 & 65.4 & 33.7 & 1.56 & 36.9 \\
Sephadex G-100 chromatography & 1403 & 6.9 & 202.6 & 9.38 & 23.5 \\
\hline
\end{tabular}

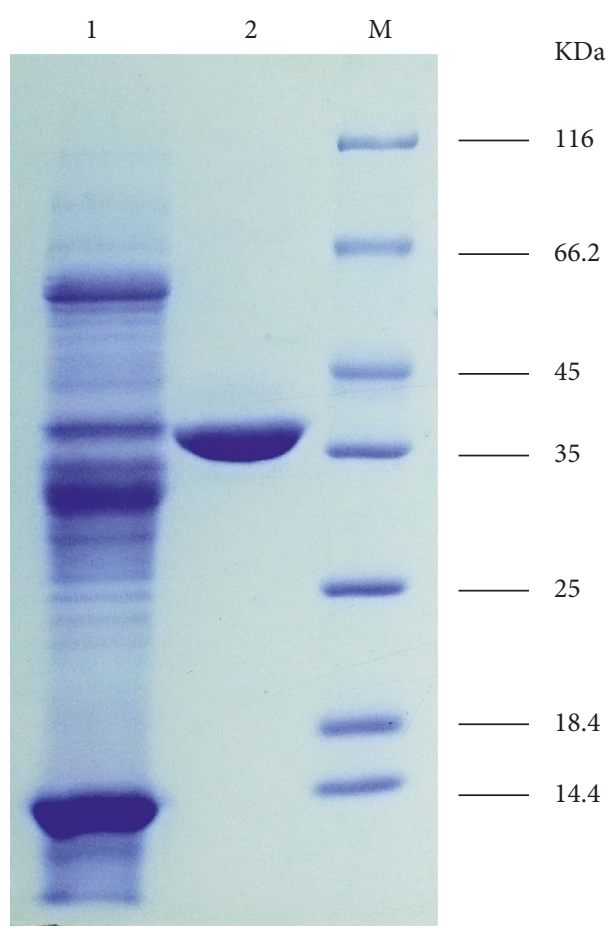

Figure 1: Molecular weight analysis of purified L-ASP obtained from P. carotovorum using SDS-PAGE on 12.5\% gel of stained with coomassie blue; M-Fermentas ladder; 1-crude extract of L-ASP; 2-purified L-ASP.

TABLE 2: Determined physiochemical characteristics of liposomes loaded L-ASP.

\begin{tabular}{lcccc}
\hline Samples & Size $(\mathrm{nm})$ & PDI & Zeta potential $(\mathrm{mV})$ & EE $(\%)$ \\
\hline Blank liposome $^{*}$ & $97.53 \pm 22.17$ & $0.24 \pm 0.02$ & $-22.80 \pm 0.00$ & - \\
L-ASP-liposome & $93.03 \pm 0.49$ & $0.22 \pm 0.02$ & $-15.45 \pm 6.72$ & $53.99 \pm 5.44$ \\
\hline
\end{tabular}

${ }^{*}$ Blank liposome containing soy lecithin, cholesterol, and DSPE-PEG2000.

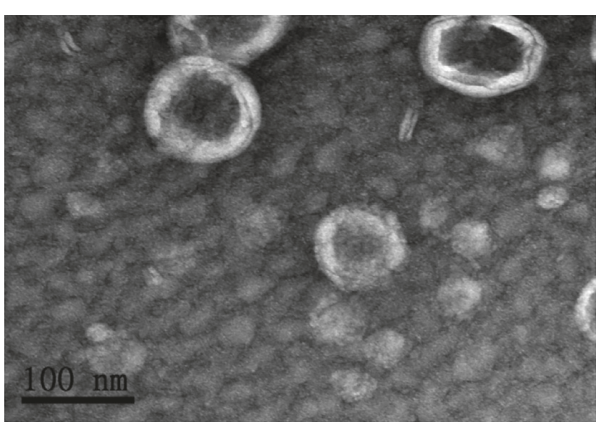

(a)

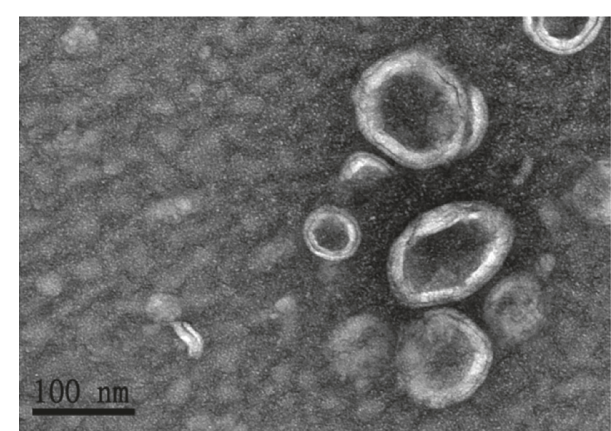

(b)

Figure 2: The TEM images of nanoliposomes (Jeol 1200EX TEM system, Japan) (a) DSPE-PEG2000-soy lecithin-cholesterol-loaded L-ASP nanoliposomes; (b) blank liposomal nanospheres. 
TABle 3: Cytotoxic activities of L-ASP-encapsulated liposomes and free L-ASP on different cell lines.

\begin{tabular}{lccccc}
\hline \multirow{2}{*}{ Samples } & & \multicolumn{3}{c}{ Values of IC $_{50}(\mathrm{UI} / \mathrm{ml})$} \\
& LLC & HepG2 & SK-LU-1 & MCF-7 & NTERA-2 \\
\hline Loaded L-ASP liposomes & $0.23 \pm 0.02$ & $0.28 \pm 0.01$ & $0.21 \pm 0.03$ & $0.36 \pm 0.05$ & $0.23 \pm 0.02$ \\
Free L-ASP & $0.079 \pm 0.001$ & $0.044 \pm 0.004$ & $0.037 \pm 0.001$ & $0.12 \pm 0.01$ & $0.038 \pm 0.003$ \\
\hline
\end{tabular}

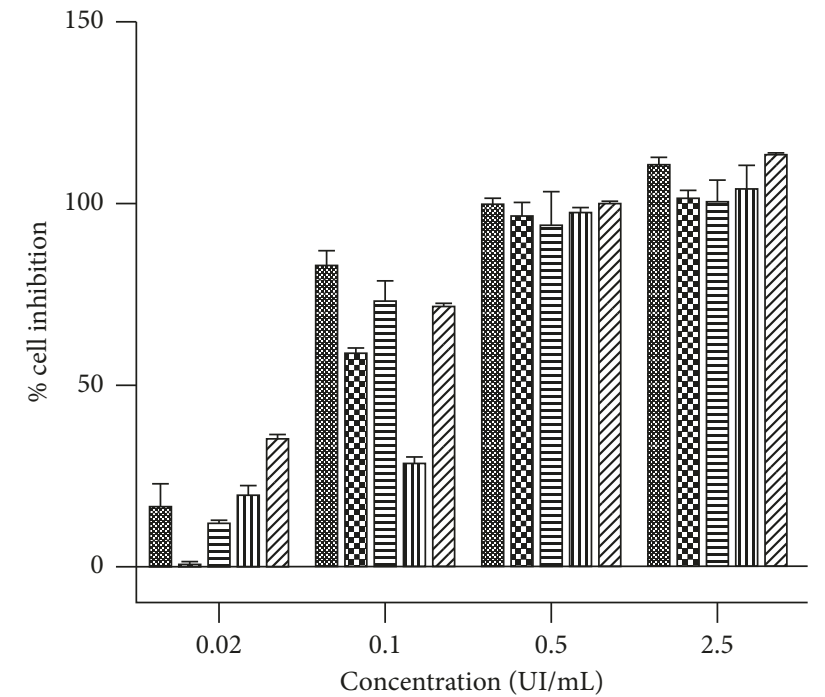

Cytotoxic effect of L-asparaginase

\begin{tabular}{|c|c|}
\hline SK-LU-1 & mس MCF-7 \\
\hline$\$$ LLC & שய NTERA-2 \\
\hline
\end{tabular}

(a)

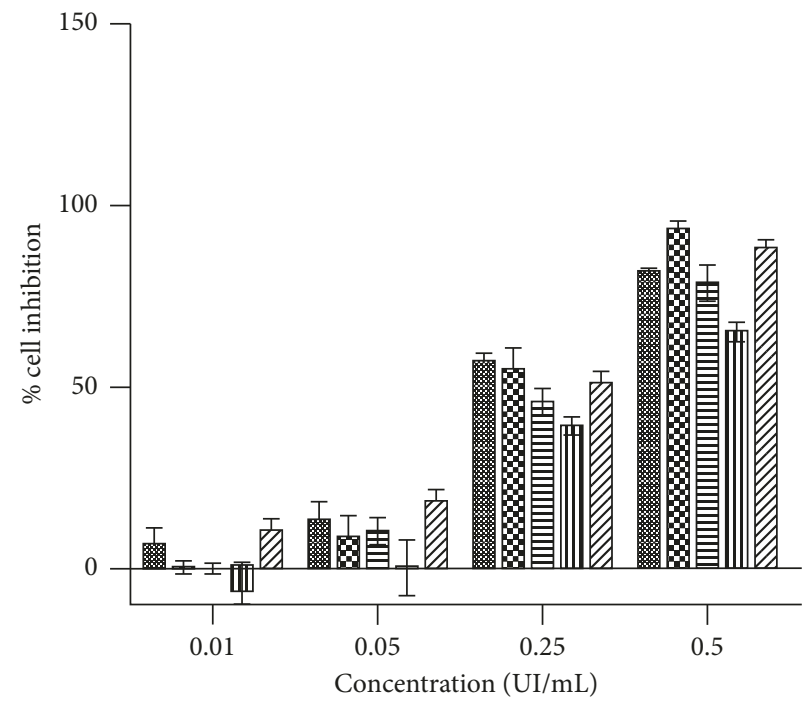

Cytotoxic effect of L-asparaginase liposome

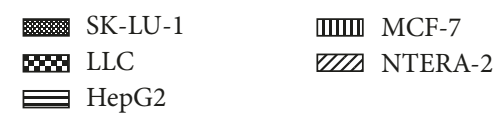

(b)

FIGURE 3: Effectiveness of free L-ASP (a) and L-ASP-encapsulated liposomes (b) on different cell lines. Cultured cells $\left(1 \times 10^{4}\right.$ cells/well) were treated with different concentrations of either liposomal (a) or free L-ASP (b). Normal saline was served as the negative control. Each value represents the mean $\pm \mathrm{SD}$.

[17]. The reduction in L-ASP cytotoxicity with liposomal encapsulation might be due to the slower release rate and slower substrate depletion, allowing cells to adapt and synthesize asparagine themselves.

3.5. In Vivo Antitumor Activity of Liposomal L-ASP. The antitumor activity of liposome-L-ASP was examined in $\mathrm{BALB} / \mathrm{c}$ mice harboring tumors induced by LLC cells (Figure 4). Groups of mice were treated with two doses of liposomal L-ASP (6 or $3 \mathrm{UI} /$ mouse) or free L-ASP (6 UI/ mouse) intravenously. The tumor volumes of the mice were analyzed at different time points. Dose-dependent antitumor activity of liposomal L-ASP was observed. Twenty-eight days after LLC inoculation, the tumors of the group treated with $6 \mathrm{UI}$ liposomal L-ASP measured $6309.11 \pm 414.06 \mathrm{~mm}^{3}$, which was significantly smaller than in the negative control $\left(9,319.35 \pm 469.58 \mathrm{~mm}^{3}, P<0.01\right)$. Liposomal L-ASP suppressed tumor growth more strongly and significantly at a dose of 6 UI than at a dose of 3 UI $\left(7,885.80 \pm 824.36 \mathrm{~mm}^{3}\right)(P<0.05)$. The tumors in the group treated with free L-ASP $\left(7,544.94 \pm 284.05 \mathrm{~mm}^{3}\right)$ were larger than those in the group treated with liposomal L-ASP at the same dose (6 UI/mouse), and this difference was significant $(P<0.05)$ (Figure 4$)$. The mice treated with $6 \mathrm{UI} /$ mouse untrapped L-ASP showed smaller volumes of tumors in comparison with the $3 \mathrm{UI} /$ mouse liposomal treated group but no significance was observed $(P>0.05)$. The mean body weight of the mice in the liposomal L-ASP groups was similar to that of the negative controls at the end of the experiment, indicating that the encapsulation did not induce toxicity (data not shown).

Furthermore, the administration of liposomal L-ASP prolonged the lives of the mice (Table 4). The median survival of mice treated with 6 UI liposomal L-ASP was $35.00 \pm 1.13$ days, which was $12.30 \%$ longer than that of control mice $(P<0.05)$. The life span also increased slightly in the free L-ASP group, but not significantly.

Since the early 1970s, L-ASP has been used to treat ALL. Currently, L-ASP is also used to treat ovarian cancer, hepatocellular carcinoma, and gastric carcinoma [31]. However, L-ASP can induce fever, thrombosis, and impaired liver, kidney, and central nervous system function, which may be related to the glutaminase activity of L-ASP [32]. 


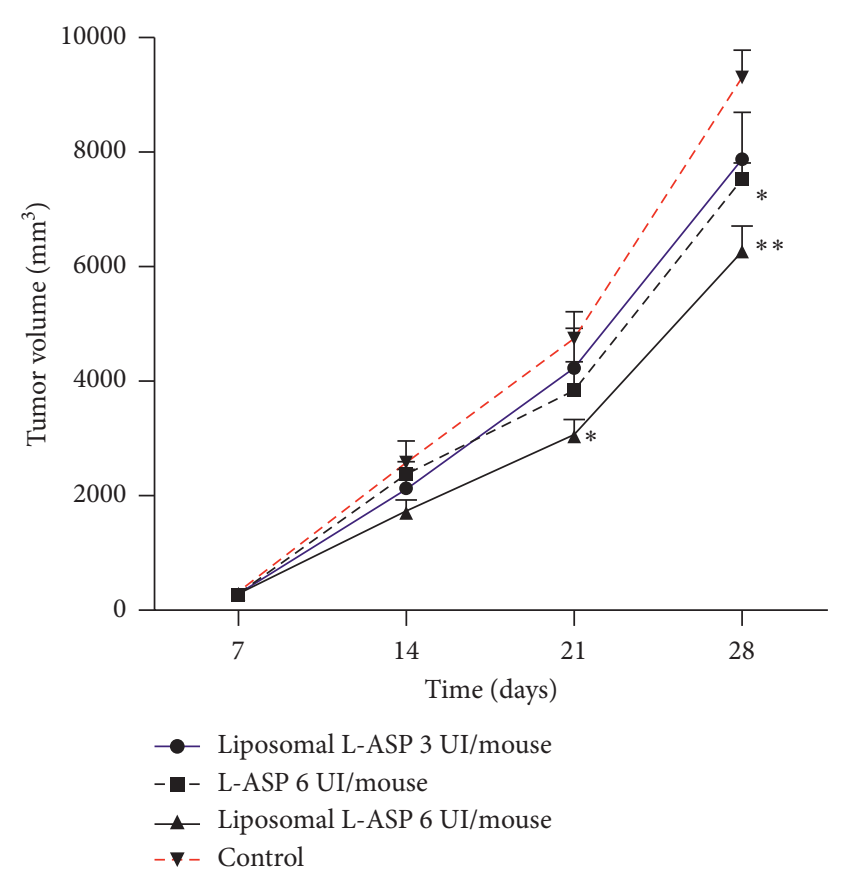

Figure 4: Tumor growth after treatment with free L-ASP or liposomal L-ASP. BALB/c mice harboring tumors induced by LLC cells were treated with either free L-ASP (6 UI/mouse) or encapsulated LASP liposomes at either $6 \mathrm{UI} / \mathrm{mouse}$ or $3 \mathrm{UI} /$ mouse $(n=6)$. Liposome-entrapped L-ASP at both doses $6 \mathrm{UI} /$ mouse and $3 \mathrm{UI} /$ mouse significantly inhibited tumor growth after 28 days compared with the negative control (blank liposome) $\left({ }^{* *} P<0.01\right.$ and ${ }^{*} P<0.05$, respectively). Error bars represent standard error (SE).

TABLE 4: Effects of liposome-L-ASP and free L-ASP on survival time of LLC cells bearing mice from different experimented groups (mean \pm SE) $(n=6)$.

\begin{tabular}{|c|c|c|}
\hline Groups & $\begin{array}{l}\text { Mean survival time } \\
\text { (days) }\end{array}$ & $\%$ ILS \\
\hline $\begin{array}{l}\text { Control group (blank } \\
\text { lipsosome) }\end{array}$ & $31.17 \pm 1.28$ & - \\
\hline Liposomal-L-ASP (3 UI/mouse) & $33.33 \pm 0.92$ & 6.95 \\
\hline Liposomal-L-ASP (6 UI/mouse) & $35.00 \pm 1.13^{*}$ & 12.30 \\
\hline Free L-ASP (6 UI/mouse) & $32.17 \pm 0.79$ & 3.21 \\
\hline
\end{tabular}

According to Kumar et al. [15], L-ASP from Pectobacterium carotovorum is free of glutaminase activity, which might avoid the disadvantages of L-ASP isolated from bacteria such as Escherichia coli and Erwinia chrysanthemi. In our study, both free and liposomal L-ASP isolated from P. carotovorum showed strong activity against various solid cancer cell lines, including lung, liver, and breast carcinomas and pluripotent human embryonic carcinoma cells. Shiromizu et al. reported similar activity of L-ASP in colon cancer (C-26), a murine sarcoma cell line (S-180), and murine breast cancer (4T1) [13].

Other limitations of L-ASP treatment include its short biological half-life $\left(T_{50}=2.88 \mathrm{~h}\right)$, which necessitates increasing the dosage and intervals of L-ASP treatment [33]. Liposomes are considered an effective solution to these obstacles. Since their discovery in the 1960s, liposomes have been used as drug carriers to enhance the potency and to reduce the toxicity of therapeutic agents. Liposomes also improve the delivery of therapeutic agents to specific sites in the body $[18,34]$. As reported elsewhere, solid tumors show enhanced permeability by and retention of lipids and macromolecules $[35,36]$. Some effects in some solid tumors are not observed in normal tissues or organs, such as extensive angiogenesis and impaired lymphatic drainage/ recovery [37]. These effects lead to higher delivery of a liposome-encapsulated drug to tumors compared with other sites. However, the size of liposomes affects the circulation of drug-loaded liposomes, especially liposomal L-ASP. Larger liposomes are easily opsonized and then rapidly removed from the blood by the mononuclear phagocytic system [38], whereas small liposomes (range $50-200 \mathrm{~nm}$ ) increase the circulation time of loaded molecules, such as enzymes [39]. With the thin film method, simplified dehydrationrehydration vesicles produce large liposomes. However, our use of bath sonication produced liposomes smaller than $200 \mathrm{~nm}$. This suitable average size helps to prolong the retention time of circulating liposomal L-ASP in vivo and its accumulation in tumors.

Additionally, the PEGylation of the liposomes helped them to avoid recognition by the mononuclear phagocytic system. Other outstanding features of PEG-coated liposomes include avoiding aggregation between liposomal particles and reduced clearance and immunogenicity [40]. These result in higher antitumor efficacy of liposomal L-ASP in vivo. However, the drug release rates of PEGylated formulations were lower than those with no PEGylation [41, 42], which may be an additional explanation for the lower in vitro cytotoxic activity of liposomal L-ASP.

\section{Conclusion}

Using the dehydrate-rehydrate thin film method, L-ASP was effectively encapsulated into liposome carriers at an efficiency of $53.99 \pm 5.44 \%$. PEGylated nanoliposomal L-ASP first showed significantly improved antitumor activities compared to those of free L-ASP $(P<0.05)$ in LLC induced $\mathrm{BALB} / \mathrm{c}$ mice but showed lower activity in an in vitro assay. Our PEGylated nanoliposomal L-ASP exhibited improved pharmacological properties and bioavailability and should be considered for further clinical applications against carcinoma tumors.

\section{Data Availability}

The physicochemical data of blank and liposomal L-ASP used to support the findings of this study are included within the supplementary information files.

\section{Conflicts of Interest}

The authors report no conflicts of interest.

\section{Acknowledgments}

The authors acknowledge the financial support from Vietnam Academy of Science and Technology [1] under the 
grants VAST04.10/18-19 and VAST.HTQT.PHAP.02/1718.

\section{Supplementary Materials}

Supplementary Table 1: concentration of L-ASP in nanoliposomes. Supplementary Table 2: relative concentration of L-ASP with concentration of liposomes in Figure 3(B). Supplementary Figure 1: normalized tumor volume change to body weight after treatment with free L-ASP or liposomal L-ASP at different times. BALB/c mice harboring tumors induced by LLC cells were treated with either free L-ASP (6 UI/mouse) or encapsulated L-ASP liposomes at either $6 \mathrm{UI} / \mathrm{mouse}$ or $3 \mathrm{UI} / \mathrm{mouse}(n=6)$. Liposome-entrapped L-ASP at both doses $6 \mathrm{UI} /$ mouse and $3 \mathrm{UI} /$ mouse significantly inhibited tumor growth after 28 days compared with the negative control (blank liposome) $\left({ }^{* *} P<0.01\right.$ and ${ }^{*} P<0.05$, respectively). Error bars represent standard error (SE). (Supplementary Materials)

\section{References}

[1] A. Shrivastava, A. A. Khan, M. Khurshid, M. A. Kalam, S. K. Jain, and P. K. Singhal, "Recent developments in L-Asparaginase discovery and its potential as anticancer agent," Critical Reviews in Oncology/Hematology, vol. 100, pp. 1-10, 2016.

[2] M. Amylon, J. Shuster, J. Pullen et al., "Intensive high-dose asparaginase consolidation improves survival for pediatric patients with $\mathrm{T}$ cell acute lymphoblastic leukemia and advanced stage lymphoblastic lymphoma: a pediatric oncology group study," Leukemia, vol. 13, no. 3, pp. 335-342, 1999.

[3] I. Hann, S. Richards, A. Vora et al., "Benefit of intensified treatment for all children with acute lymphoblastic leukaemia: results from MRC UKALL XI and MRC ALL97 randomised trials," Leukemia, vol. 14, no. 3, pp. 356-363, 2000.

[4] M. Schrappe, A. Reiter, W. D. Ludwig et al., "Improved outcome in childhood acute lymphoblastic leukemia despite reduced use of anthracyclines and cranial radiotherapy: results of trial ALL-BFM 90. German-Austrian-Swiss ALL-BFM Study Group," Blood, vol. 95, no. 11, pp. 3310-3322, 2000.

[5] V. J. Land, W. W. Sutow, D. J. Fernbach, D. M. Lane, and T. E. Williams, "Toxicity of L-Asparaginase in children with advanced leukemia," Cancer, vol. 30, no. 2, pp. 339-347, 1972.

[6] H. F. Oettgen, P. A. Stephenson, M. K. Schwartz et al., "Toxicity of E. coli L-Asparaginase in man," Cancer, vol. 25, no. 2, pp. 253-278, 1970.

[7] R. W. Baldwin, "Experimental and clinical effects of L-Asparaginase," Immunology, vol. 21, no. 4, p. 727, 1971.

[8] R. G. Peterson, R. E. Handschumacher, and M. S. Mitchell, "Immunological responses to L-Asparaginase," Journal of Clinical Investigation, vol. 50, no. 5, pp. 1080-1090, 1971.

[9] H. Daraee, A. Etemadi, M. Kouhi, S. Alimirzalu, and A. Akbarzadeh, "Application of liposomes in medicine and drug delivery," Artificial Cells, Nanomedicine, and Biotechnology, vol. 44, no. 1, pp. 381-391, 2016.

[10] J. Li, X. Wang, T. Zhang et al., "A review on phospholipids and their main applications in drug delivery systems," Asian Journal of Pharmaceutical Sciences, vol. 10, no. 2, pp. 81-98, 2015.

[11] J. O. C. S. Jorge, R. Perez-Soler, J. G. Morais, and M. E. N. M. Cruz, "Liposomal palmitoyl-L-Asparaginase: characterization and biological activity," Cancer Chemotherapy and Pharmacology, vol. 34, no. 3, pp. 230-234, 1994.

[12] M. Jahadi and K. Khosravi-Darani, "Liposomal encapsulation enzymes: from medical applications to kinetic characteristics," Mini-Reviews in Medicinal Chemistry, vol. 17, no. 4, pp. 366-370, 2017.

[13] S. Shiromizu, N. Kusunose, N. Matsunaga, S. Koyanagi, and S. Ohdo, "Optimizing the dosing schedule of L-Asparaginase improves its anti-tumor activity in breast tumor-bearing mice," Journal of Pharmacological Sciences, vol. 136, no. 4, pp. 228-233, 2018.

[14] R. Gulati, R. K. Saxena, and R. Gupta, "A rapid plate assay for screening L-Asparaginase producing micro-organisms," Letters in Applied Microbiology, vol. 24, no. 1, pp. 23-26, 1997.

[15] S. Kumar, V. Venkata Dasu, and K. Pakshirajan, "Purification and characterization of glutaminase-free L-Asparaginase from Pectobacterium carotovorum MTCC 1428," Bioresource Technology, vol. 102, no. 2, pp. 2077-2082, 2011.

[16] J. C. Wriston Jr., “[79] asparaginase," Glutamate, Glutamine, Glutathione, and Related Compounds, vol. 113, pp. 608-618, 1985.

[17] M. E. M. Cruz, M. M. Gaspar, F. Lopes, J. S. Jorge, and R. Perez-Soler, "Liposomal L-Asparaginase: in vitro evaluation," International Journal of Pharmaceutics, vol. 96, no. 1-3, pp. 67-77, 1993.

[18] P. R. Kulkarni, J. D. Yadav, and K. A. Vaidya, "Liposomes: a novel drug delivery system," International Journal of Current Pharmaceutical Research, vol. 3, no. 2, pp. 10-18, 2011.

[19] D. A. Scudiero, R. H. Shoemaker, K. D. Paull et al., "Evaluation of a soluble tetrazolium/formazan assay for cell growth and drug sensitivity in culture using human and other tumor cell lines," Cancer Research, vol. 48, no. 17, pp. 4827-4833, 1988.

[20] M. M. Jensen, J. T. Jørgensen, T. Binderup, and A. Kjaer, "Tumor volume in subcutaneous mouse xenografts measured by microCT is more accurate and reproducible than determined by $18 \mathrm{~F}-\mathrm{FDG}$-microPET or external caliper," BMC Medical Imaging, vol. 8, no. 1, 2008.

[21] A. Akbarzadeh, R. Rezaei-Sadabady, S. Davaran et al., "Liposome: classification, preparation, and applications," Nanoscale Research Letters, vol. 8, no. 1, p. 102, 2013.

[22] P. van Hoogevest and A. Wendel, "The use of natural and synthetic phospholipids as pharmaceutical excipients," European Journal of Lipid Science and Technology, vol. 116, no. 9, pp. 1088-1107, 2014.

[23] O. Nag and V. Awasthi, "Surface engineering of liposomes for stealth behavior," Pharmaceutics, vol. 5, no. 4, pp. 542-569, 2013.

[24] M.-L. Briuglia, C. Rotella, A. McFarlane, and D. A. Lamprou, "Influence of cholesterol on liposome stability and on in vitro drug release," Drug Delivery and Translational Research, vol. 5, no. 3, pp. 231-242, 2015.

[25] S. Honary and F. Zahir, "Effect of zeta potential on the properties of nano-drug delivery systems-a review (part 2)," Tropical Journal of Pharmaceutical Research, vol. 12, no. 2, pp. 265-273, 2013.

[26] S. Salgin, U. Salgin, and S. Bahadir, "Zeta potentials and isoelectric points of biomolecules: the effects of ion types and ionic strengths," International Journal of Electrochemical Science, vol. 7, no. 12, pp. 12404-12414, 2012.

[27] Y. Zhang, M. Yang, N. G. Portney et al., "Zeta potential: a surface electrical characteristic to probe the interaction of nanoparticles with normal and cancer human breast epithelial 
cells," Biomedical Microdevices, vol. 10, no. 2, pp. 321-328, 2008.

[28] E. Bahreini, K. Aghaiypour, R. Abbasalipourkabir, A. Mokarram, M. Goodarzi, and M. Saidijam, "Preparation and nanoencapsulation of L-Asparaginase II in chitosantripolyphosphate nanoparticles and in vitro release study," Nanoscale Research Letters, vol. 9, no. 1, p. 340, 2014.

[29] A. S. Krall, S. Xu, T. G. Graeber, D. Braas, and H. R. Christofk, "Asparagine promotes cancer cell proliferation through use as an amino acid exchange factor," Nature Communications, vol. 7, no. 1, p. 11457, 2016.

[30] S. A. Moharib, "Anticancer activity of L-Asparaginase produced from vigna unguiculata," World Scientific Research, vol. 5, no. 1, pp. 1-12, 2018.

[31] D. Covini, S. Tardito, O. Bussolati et al., "Expanding targets for a metabolic therapy of cancer: L-Asparaginase," Recent Patents on Anti-Cancer Drug Discovery, vol. 7, no. 1, pp. 4-13, 2012.

[32] L. Huang, Y. Liu, Y. Sun, Q. Yan, and Z. Jiang, "Biochemical characterization of a novel L-Asparaginase with low glutaminase activity from Rhizomucor miehei and its application in food safety and leukemia treatment," Applied and Environmental Microbiology, vol. 80, no. 5, pp. 1561-1569, 2014.

[33] A. De and D. N. Venkatesh, "Design and evaluation of liposomal delivery system for L-Asparaginese," Journal of Applied Pharmaceutical Science, vol. 2, no. 8, p. 112, 2012.

[34] A. Babu, A. K. Templeton, A. Munshi, and R. Ramesh, "Nanodrug delivery systems: a promising technology for detection, diagnosis, and treatment of cancer," AAPS PharmSciTech, vol. 15, no. 3, pp. 709-721, 2014.

[35] H. Kobayashi, R. Watanabe, and P. L. Choyke, "Improving conventional enhanced permeability and retention (EPR) effects; what is the appropriate target," Theranostics, vol. 4, no. 1, pp. 81-89, 2013.

[36] A. D. Wong, M. Ye, M. B. Ulmschneider, and P. C. Searson, "Quantitative analysis of the enhanced permeation and retention (EPR) effect," PloS One, vol. 10, no. 5, Article ID e0123461, 2015.

[37] S. Stapleton, M. Milosevic, C. Allen et al., "A mathematical model of the enhanced permeability and retention effect for liposome transport in solid tumors," PloS One, vol. 8, no. 12, Article ID e81157, 2013.

[38] A. C. Anselmo, V. Gupta, B. J. Zern et al., "Delivering nanoparticles to lungs while avoiding liver and spleen through adsorption on red blood cells," ACS Nano, vol. 7, no. 12, pp. 11129-11137, 2013.

[39] M. M. Gaspar, R. Perez-Soler, and M. E. M. Cruz, "Biological characterization of L-Asparaginase liposomal formulations," Cancer Chemotherapy and Pharmacology, vol. 38, no. 4, pp. 373-377, 1996.

[40] M. L. Immordino, F. Dosio, and L. Cattel, "Stealth liposomes: review of the basic science, rationale, and clinical applications, existing and potential," International Journal of Nanomedicine, vol. 1, no. 3, pp. 297-315, 2006.

[41] F. Atyabi, A. Farkhondehfai, F. Esmaeili, and R. Dinarvand, "Preparation of pegylated nano-liposomal formulation containing SN-38: in vitro characterization and in vivo biodistribution in mice," Acta Pharmaceutica, vol. 59, no. 2, pp. 133-144, 2009.

[42] L. Cai, X. Wang, W. Wang et al., "Peptide ligand and PEGmediated long-circulating liposome targeted to FGFR overexpressing tumor in vivo," International Journal of Nanomedicine, vol. 7, pp. 4499-4510, 2012. 


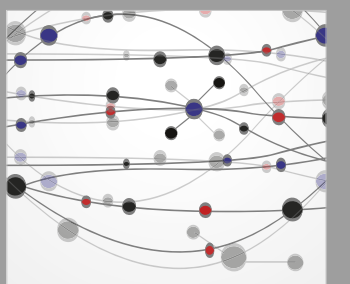

The Scientific World Journal
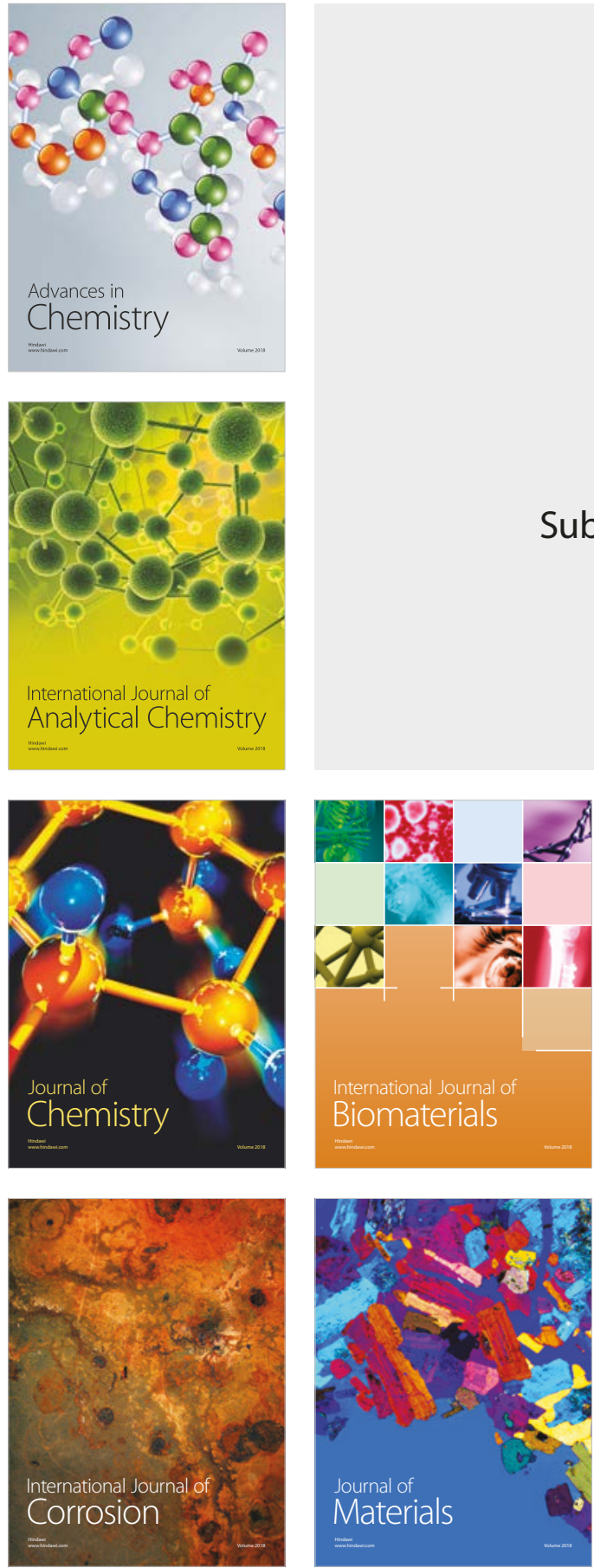

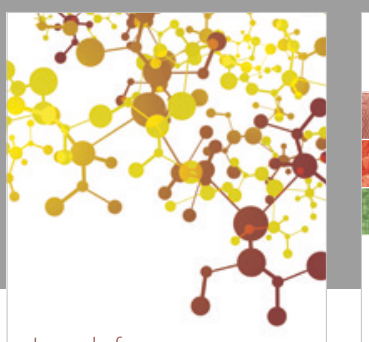

Journal of

Applied Chemistry
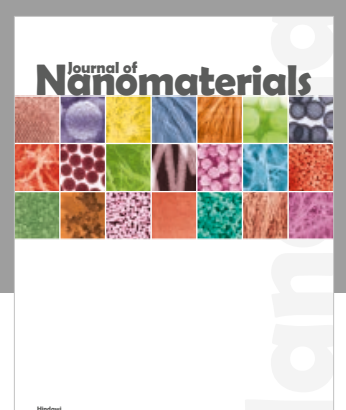

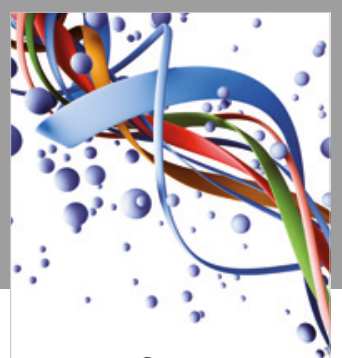

Scientifica

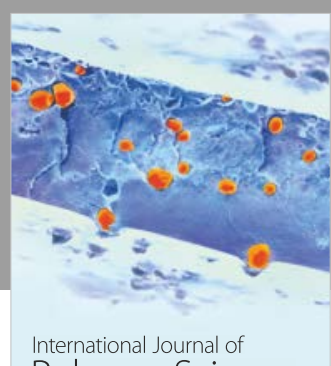

Polymer Science

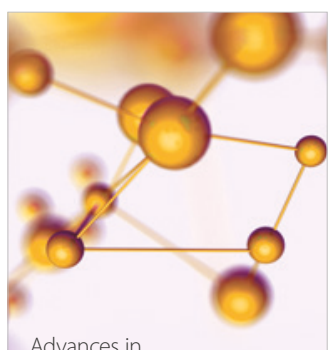

Physical Chemistry
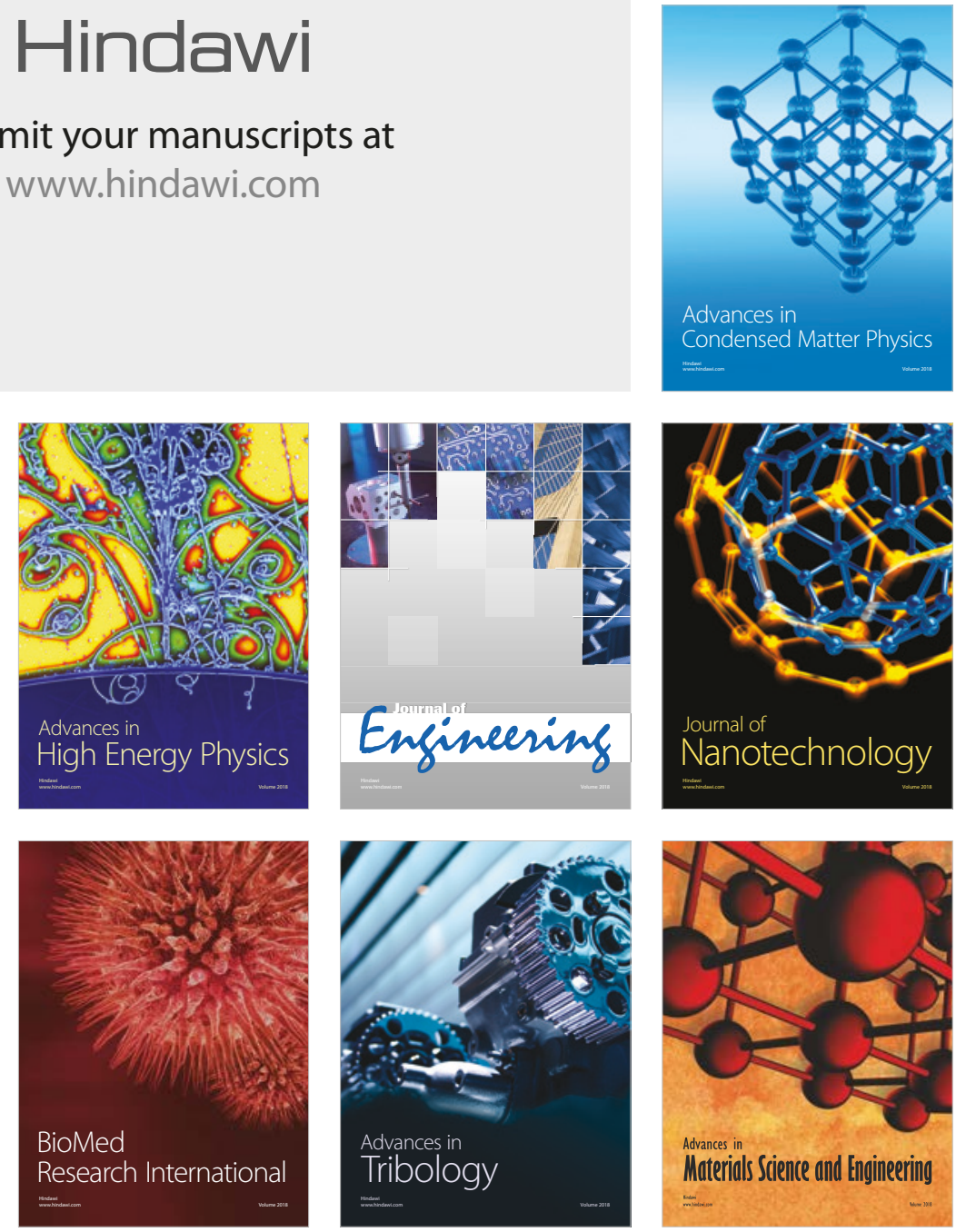